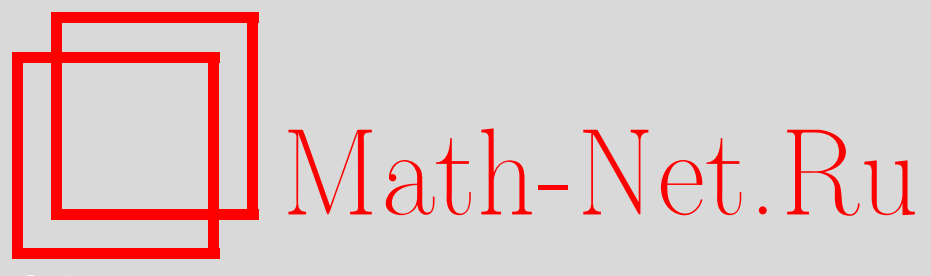

И. А. Иванишко, В. Г. Кротов, Компактность вложений соболевского типа на метрических пространствах с мерой, Матем. заметки, 2009, том 86, выпуск 6, 829-844

DOI: https://doi.org/10.4213/mzm8526

Использование Общероссийского математического портала Math-Net.Ru подразумевает, что вы прочитали и согласны с пользовательским соглашением http://www . mathnet.ru/rus/agreement

Параметры загрузки:

IP : 54.198 .55 .26

26 апреля 2023 г., 15:26:51

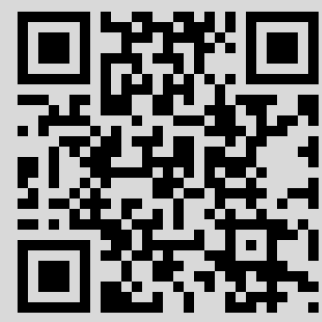


Том 86 выпуск 6 декабрь 2009

\section{Компактность вложений соболевского типа на метрических пространствах с мерой}

\section{И. А. Иванишко, В. Г. Кротов}

Установлены условия компактности вложений некоторых классов функций на метрическом пространстве с мерой, удовлетворяющей условию удвоения. Эти классы определяются в терминах $L^{p}$-суммируемости максимальных функций, измеряющих локальную гладкость.

Библиография: 22 названия.

1. Введение. Пусть $X$ - метрическое пространство с метрикой $d$ и регулярной борелевской мерой $\mu$. На протяжении всей работы мы будем систематически пользоваться следующими обозначениями:

$$
B(x, r)=\{y \in X: d(x, y)<r\}
$$

для шара с центром в точке $x \in X$ радиуса $r>0$ и

$$
f_{B}=f_{B} f d \mu=\frac{1}{\mu B} \int_{B} f d \mu
$$

для среднего значения функции $f \in L^{1}(B)$ по шару $B \subset X$. Кроме того, через $L^{p}(X), p \geqslant 1$, обозначаем множество (классов эквивалентности) измеримых функций, для которых конечна норма

$$
\|f\|_{L^{p}(X)}=\left(\int_{X}|f|^{p} d \mu\right)^{1 / p} .
$$

Мы предполагаем, что мера $\mu$ удовлетворяет условию удвоения: существует постоянная $c_{\mu}>0$ такая, что

$$
\mu B(x, 2 r) \leqslant c_{\mu} \mu B(x, r), \quad x \in X, \quad r>0 .
$$

В таком случае $(X, d, \mu)$ обычно называют пространством однородного типа [1].

Введем еще классы функциональных параметров. Для $a>0$ обозначим через $\Omega(a)$ класс возрастающих функций $\eta:(0,1] \rightarrow(0,+\infty)$, для которых $\eta(+0)=0$ и $\eta(r) r^{-a}$ убывает. Кроме этого, положим

$$
\Omega=\bigcup_{a>0} \Omega(a)
$$

(C) И. А. Иванишко, В. Г. Кротов, 2009 
Для $\eta \in \Omega$ определим максимальные операторы

$$
\mathscr{N}_{\eta} f(x)=\sup _{B(y, r) \ni x} \frac{1}{\eta(r)} f_{B(y, r)}|f(y)-f(x)| d \mu(y),
$$

где точная верхняя грань берется по всем шарам $B(y, r)$ радиуса $0<r \leqslant 1$, содержащим точку $x \in X$. Значения функции понимаются как $\lim _{r \rightarrow+0} f_{B(x, r)}$, который существует почти всюду для любой функции $f \in L_{\mathrm{loc}}^{1}(X)$ (см. [2; с. 4]).

Впервые такие максимальные функции появились в работах Кальдерона [3] и Кальдерона и Скотта [4] в случае $X=\mathbb{R}^{n}$ для $\eta(r)=r^{\alpha}, 0<\alpha \leqslant 1$. В [3], [4] рассматривались также более общие операторы, когда $\alpha>0$ и в определении $\mathscr{N}_{\eta}$ вместо $f(x)$ вычитается некоторый многочлен, степень которого зависит от $\alpha$. Систематическому изучению максимальных функций такого сорта на $\mathbb{R}^{n}$ посвящена монография [5].

Для любой функции $\eta \in \Omega(1)$ операторы $\mathscr{N}_{\eta}$ в случае $X=[0,1]^{n}$ впервые изучал Коляда [6], [7]. Мотивировкой для рассмотрения такого спектра характеристик $\eta$ было расширение возможностей для более тонкой классификации функций по их локальной гладкости - как показывает следующее простое неравенство:

$$
|f(x)-f(y)| \leqslant \eta(d(x, y))\left[\mathscr{N}_{\eta} f(x)+\mathscr{N}_{\eta} f(y)\right]
$$

$\mathscr{N}_{\eta} f$ измеряет локальную гладкость функции $f$.

Отметим также, что в неявном виде максимальные операторы $\mathscr{N}_{\eta}$ встречались также в работе Осколкова [8] при изучении задачи П. Л. Ульянова о количественных оценках $C$-свойства Лузина для функций с заданной мажорантой $L^{p}$-модуля непрерывности.

Для $\eta \in \Omega$ рассмотрим классы

$$
C_{\eta}^{p}(X)=\left\{f \in L^{p}(X):\|f\|_{C_{\eta}^{p}}=\|f\|_{L^{p}(X)}+\left\|\mathscr{N}_{\eta} f\right\|_{L^{p}(X)}<\infty\right\} .
$$

ЗАмечание 1 . В случае $\eta(r)=r$ и $X=\mathbb{R}^{n}$ класс $C_{\eta}^{p}\left(\mathbb{R}^{n}\right)$ совпадает с классическим пространством Соболева $W_{1}^{p}\left(\mathbb{R}^{n}\right)$ (Кальдерон [3], см. также [5]).

Если $X$ - произвольное метрическое пространство и $\eta(r)=r$, то $C_{\eta}^{p}(X)$ совпадает с пространством Хайлаша-Соболева [9].

Для классов $C_{\eta}^{p}(X)$ справедлива следующая теорема вложения, в которой предполагается, что $X$ ограничено.

TeOPEma 1. Пусть $, 1 \leqslant p<q<\infty, \sigma \in \Omega$,

$$
\eta(r)=\sigma(r) r^{\gamma(1 / p-1 / q)} .
$$

Тогда $a^{1}$

$$
\left\|\mathscr{N}_{\sigma} f\right\|_{L^{q}(X)} \leqslant c\left\|\mathscr{N}_{\eta} f\right\|_{L^{p}(X)} .
$$

В частности, $C_{\eta}^{p}(X) \subset C_{\sigma}^{q}(X)$.

\footnotetext{
13десь и всюду ниже через $c$ мы обозначаем различные положительные постоянные, зависящие, возможно, лишь от параметров $p, q, \eta, \sigma$ и т.д., определяющих рассматриваемые пространства функций.
} 
В теореме 1 участвует показатель $\gamma>0$ из количественной формы условия удвоения (2.1), который выполняет роль "размерности" пространства $X$; если $X=\mathbb{R}^{n}$, то $\gamma=n$.

В случае $X=\mathbb{R}^{n}$ для степенных функций $\eta(r)=r^{\alpha}$ при $0<\alpha \leqslant 1$ и $\sigma(r)=r^{\beta}$, $\beta=\alpha-n(1 / p-1 / q)$ утверждение теоремы 1 доказано в работе [4].

Для $X=[0,1]^{n}, n \geqslant 1$, теорема 1 доказана Колядой [6], [7]. В [7] показано также, что условие (1.5) в теореме 1 является неулучшаемым. Общий вариант теоремы 1 доказал первый автор [10], используя метод работы $[7]^{2}$.

Целью нашей работы является нахождение условий для компактности вложений (1.6) и $C_{\eta}^{p}(X) \subset L^{q}(X)$ - они будут рассмотрены в п. 4 . Кроме того, в п. 3 будет показано, что с помощью функций $\mathscr{N}_{\eta}, \eta \in \Omega$, можно получить полную классификацию функций из $L^{p}(X)$.

Отметим, что в формулировках теоремы 1 в работах [7], [10] вместо условия $\sigma \in \Omega$ (см. (1.2)) фигурировало $\eta, \sigma \in \Omega(1)$, но на самом деле в доказательствах использовалось лишь то, что $\sigma \in \Omega$.

ЗАмЕчаниЕ 2 . Если $X=\mathbb{R}^{n}$ или $X=[0,1]^{n}$ с евклидовой метрикой и мерой Лебега, то в определении классов $C_{\eta}^{p}(X)$ естественно рассматривать только функции $\eta \in \Omega(1)$. Причиной для этого является то, что при условии

$$
\lim _{r \rightarrow+0} \frac{\eta(r)}{r}=0
$$

любая функция $f \in C_{\eta}^{p}(X)$ будет эквивалентна постоянной.

Утверждение из замечания 2 можно доказать следующим образом. Пусть, например, $X=\mathbb{R}^{n}$ и функция $f \in C_{\eta}^{p}\left(\mathbb{R}^{n}\right)$. Так как при условии (1.7) класс $C_{\eta}^{p}\left(\mathbb{R}^{n}\right)$ содержится в обычном соболевском пространстве $W_{1}^{p}\left(\mathbb{R}^{n}\right)$ (см. замечание 1 ), то $f$ имеет обобщенные производные $\partial f / \partial x_{k} \in L^{p}\left(\mathbb{R}^{n}\right), k=1, \ldots, n$, и для почти всех $x \in \mathbb{R}^{n}$ выполнено соотношение

$$
\lim _{r \rightarrow+0} \frac{1}{r} f_{B(x, r)}\left|f(y)-f(x)-\sum_{k=1}^{n} \frac{\partial f}{\partial x_{k}}(x)\left(y_{k}-x_{k}\right)\right| d y=0
$$

(см., например, $\left[11 ;\right.$ с. 176]). С другой стороны, так как $f \in C_{\eta}^{p}\left(\mathbb{R}^{n}\right)$ и выполнено (1.7), то для почти всех $x \in \mathbb{R}^{n}$

$$
\lim _{r \rightarrow+0} \frac{1}{r} f_{B(x, r)}|f(y)-f(x)| d y=0 .
$$

Отсюда легко вывести, что $\left(\partial f / \partial x_{k}\right)(x)=0$ для всех $k=1, \ldots, n$ и почти всех $x \in \mathbb{R}^{n}$, поэтому $f$ эквивалентна постоянной (см. [12; с. 129]).

ЗАмЕчАниЕ 3. В общем случае возможны ситуации, в которых классы $C_{\eta}^{p}(X)$ нетривиальны для некоторых функций $\eta$ со свойством (1.7). Это следует из того, что обычные классы Гёльдера

$$
H_{\alpha}(X)=\left\{f:|f(x)-f(y)| \leqslant c d^{\alpha}(x, y)\right\}
$$

\footnotetext{
${ }^{2}$ На самом деле в [7], [10] было доказано более сильное утверждение $\left\|\mathscr{N}_{\sigma} f\right\|_{q, p} \leqslant c\left\|\mathscr{N}_{\eta} f\right\|_{p}$ с нормой в пространстве Лоренца в левой части.
} 
могут быть нетривиальными для некоторых пространств $X$ и $\alpha>1$ - например, на кривой Коха это справедливо при $0<\alpha \leqslant \ln 4 / \ln 3$ [13].

Замечание 3 показывает, что в случае произвольного метрического пространства $X$ классы $C_{\eta}^{p}(X)$ естественно рассматривать для любой функции $\eta \in \Omega$.

ЗАмечание 4 . Теорема 1 справедлива также в случае $\sigma(r) \equiv 1$.

2. Основные понятия и вспомогательные утверждения. На самом деле мы будем рассматривать более общую ситуацию, чем указано во введении. Пусть $X$ - хаусдорфово пространство, топология которого задается квазиметрикой $d$. Последнее означает, что функция $d: X \times X \rightarrow[0, \infty)$ удовлетворяет всем аксиомам метрики, лишь неравенство треугольника заменяется условием: существует такая постоянная $a_{d} \geqslant 1$, что

$$
d(x, y) \leqslant a_{d}[d(x, z)+d(z, y)]
$$

для любых $x, y, z \in X$. Кроме того, семейство открытых шаров $\{B(x, r)\}$ образует базу окрестностей топологии $X$.

Далее используются обозначения $r(B)$ - радиус шара $B$ и $\mathscr{B}(x, t)$ - множество всех шаров $B$ радиуса $r(B)<t$, содержащих точку $x$. Пусть еще $\mathscr{B}(x, 1)=\mathscr{B}(x)$.

Как уже говорилось во введении, мы предполагаем выполненным условие удвоения (1.1). Ему можно придать количественный вид - при условии (1.1) для некоторого $\gamma>0$ выполнено неравенство

$$
\mu B(x, s) \leqslant c\left(\frac{s}{r}\right)^{\gamma} \mu B(x, r), \quad x \in X, \quad 0<r \leqslant s,
$$

которое получается итерацией неравенства (1.1).

ЗАмечание 5. Очевидно, что условие (2.1) тем сильнее, чем меньше $\gamma$. Однако, наименьшее $\gamma$, для которого выполнено (2.1), может не существовать - если

$$
\gamma_{0}=\inf \{\gamma: \text { выполнено }(2.1)\}
$$

то (2.1) может не выполняться с $\gamma=\gamma_{0}$.

Введем обычную максимальную функцию Харди-Литтлвуда

$$
M f(x)=\sup _{B \in \mathscr{B}(x)} f_{B}|f(y)| d \mu(y) .
$$

При $p>1$ она удовлетворяет неравенству (см. [2; с. 11])

$$
\|M f\|_{L^{p}(X)} \leqslant c\|f\|_{L^{p}(X)} .
$$

Ниже мы будем использовать следующее утверждение из работы [14].

Лемма 1. Пусть $\mu$-конечная борелевская мера на $X$ со свойством

$$
h(r)=\inf _{x \in X} \mu B(x, r)>0, \quad r>0 .
$$


Тогда ограниченное множество $K \subset L^{p}(X), p \geqslant 1$, удовлетворяющее условию

$$
\lim _{r \rightarrow+0} \sup _{f \in K} \int_{X}\left|f(x)-f_{B(x, r)}\right|^{p} d \mu=0
$$

является предкомпактным в $L^{p}(X)$.

В [14] это утверждение формулировалось для случая метрического пространства, но приведенное там доказательство проходит в значительно более общей ситуации. В частности, оно подходит и для случая квазиметрики.

Наряду с (1.3) нам понадобятся другие максимальные функции

$$
\begin{aligned}
& \mathscr{S}_{\eta} f(x)=\sup _{B \in \mathscr{B}(x)} \frac{1}{\eta(r(B))} f_{B}\left|f(y)-f_{B}\right| d \mu(y), \\
& \mathscr{R}_{\eta} f(x)=\sup _{B \in \mathscr{B}(x)} \frac{\left|f(x)-f_{B}\right|}{\eta(r(B))} .
\end{aligned}
$$

Ясно, что для любой функции $f \in L_{\mathrm{loc}}^{1}(X)$ максимальная функция $\mathscr{S}_{\eta} f$ является полунепрерывной снизу и, следовательно, измеримой. Для функций $\mathscr{N}_{\eta} f$ и $\mathscr{R}_{\eta} f$ измеримость получается немного сложнее (см. ниже лемму 2).

Операторы $\mathscr{N}_{\eta}, \mathscr{S}_{\eta}$ и $\mathscr{R}_{\eta}$ тесно связаны друг с другом - для них справедливы следующие очевидные поточечные неравенства:

$$
\begin{aligned}
\mathscr{S}_{\eta} f(x) & \leqslant 2 \mathscr{N}_{\eta} f(x), \\
\mathscr{R}_{\eta} f(x) \leqslant \mathscr{N}_{\eta} f(x) & \leqslant \mathscr{R}_{\eta} f(x)+M\left(\mathscr{R}_{\eta} f\right)(x),
\end{aligned}
$$

где $M$ - оператор Харди-Литтлвуда (2.2) и $\eta \in \Omega$.

Обратное к (2.8) неравенство

$$
\mathscr{N}_{\eta} f(x) \leqslant c \mathscr{S}_{\eta} f(x)
$$

доказано [15], [16] лишь для функций $\eta \in \Omega$, удовлетворяющих условию Бари

$$
\int_{0}^{t} \frac{\eta(s)}{s} d s \leqslant c \eta(t), \quad t>0 .
$$

Аналогично (1.4), но с $\mathscr{S}_{\eta}$ на месте $\mathscr{N}_{\eta}$, определим классы

$$
S_{\eta}^{p}(X)=\left\{f \in L^{p}(X):\|f\|_{S_{\eta}^{p}}=\|f\|_{L^{p}(X)}+\left\|\mathscr{S}_{\eta} f\right\|_{L^{p}(X)}<\infty\right\} .
$$

В работе [16] для классов $C_{\eta}^{p}(X)$ и $S_{\eta}^{p}(X)$ получено много различных описаний (см. также [17] в степенном случае $\eta(t)=t^{\alpha}$ ).

ЗАмЕчАниЕ 6. В определениях максимальных операторов (1.3), (2.6) и (2.7) можно было бы брать точную верхнюю грань по всем шарам, содержащим точку $x-$ не ограничивая их радиусы. Именно так они определялись, например, в [16].

Легко видеть, что такое изменение приведет к тем же классам $C_{\eta}^{p}(X)$ и $S_{\eta}^{p}(X)$ (см. (1.4) и (2.12)) с эквивалентными нормами. 
При условиях теоремы 1 справедливо также включение

$$
S_{\sigma}^{q}(X) \subset S_{\eta}^{p}(X)
$$

Это следует из неравенств (2.8) и (2.10), так как функция $\eta$ из (1.5) удовлетворяет условию Бари (2.11). Отметим, что в работах второго автора [18], [19] рассматривались также весовые вложения такого типа.

\section{3. Классификация функций из $L^{p}$.}

ЛЕмма 2. Для любой функции $f \in L_{\text {loc }}^{1}(X)$ максимальные функции (1.3) и (2.7) н-измеримы.

ДокАЗАТЕЛЬСтво. Достаточно доказать измеримость функции (2.7) на любом шаре $B_{0} \subset X$. По теореме Лузина для любого $n \in \mathbb{N}$ существует такое измеримое множество $E_{n} \subset B_{0}$, что $\mu\left(B_{0} \backslash E_{n}\right)<2^{-n}$ и сужение $\left.f\right|_{E_{n}}$ непрерывно на $E_{n}$.

Зададим $\lambda>0$ и покажем, что множество

$$
E_{n}^{\lambda}=\left\{\mathscr{R}_{\eta} f>\lambda\right\} \cap E_{n}
$$

является измеримым. Для этого возьмем точку $x \in E_{n}^{\lambda}$, найдем шар $B \in \mathscr{B}(x)$ со свойством $\left|f(x)-f_{B}\right|>\lambda \eta(r(B))$, положим

$$
\varepsilon=\frac{\left|f(x)-f_{B}\right|}{\eta(r(B))}-\lambda>0
$$

и определим $\delta>0$ так, чтобы $B(x, \delta) \subset B$ и

$$
\frac{|f(x)-f(y)|}{\eta(r(B))}<\varepsilon \quad \text { при } \quad y \in B(x, \delta) \cap E_{n} .
$$

Тогда при $y \in B(x, \delta) \cap E_{n}$ выполнено неравенство

$$
\frac{\left|f(y)-f_{B}\right|}{\eta(r(B))} \geqslant \frac{\left|f(x)-f_{B}\right|}{\eta(r(B))}-\frac{|f(x)-f(y)|}{\eta(r(B))}>(\lambda+\varepsilon)-\varepsilon=\lambda .
$$

Следовательно, $B(x, \delta) \cap E_{n} \subset E_{n}^{\lambda}$. Это означает, что $E_{n}^{\lambda}$ является пересечением $E_{n}$ и некоторого открытого множества и потому измеримо. Осталось заметить, что если

$$
E=B_{0} \backslash \bigcup_{n=1}^{\infty} E_{n}
$$

то $\mu E=0$ и

$$
\left\{\mathscr{R}_{\eta} f>\lambda\right\}=\left(\bigcup_{n=1}^{\infty} E_{n}^{\lambda}\right) \cup\left(E \cap\left\{\mathscr{R}_{\eta} f>\lambda\right\}\right),
$$

причем все множества справа измеримы.

Это же рассуждение подходит и для максимальной функции (1.3).

Отметим, что в доказательстве этой леммы условие удвоения (1.1) не используется. 
Tеорема 2. Пусть $p>1 u f \in L^{p}(X)$. Тогда $\mathscr{N}_{\eta} f \in L^{p}(X)$ для некоторой функиии $\eta \in \Omega$.

ДоказАТЕЛЬство. Можно считать, что $f$ не эквивалентна постоянной. Введем обозначение

$$
g(x, t)=\sup _{B \in \mathscr{B}(x, t)} f_{B}|f(x)-f(y)| d \mu(y), \quad 0<t \leqslant \operatorname{diam} X .
$$

Тогда выполнены условия

1) $0 \leqslant g\left(x, t_{1}\right) \leqslant g\left(x, t_{2}\right)$ при $0<t_{1}<t_{2} \leqslant \operatorname{diam} X$;

2) $\lim _{t \rightarrow+0} g(x, t)=0$ почти всюду;

3) $g \in L^{p}(X)$, где $g(x)=g(x, \operatorname{diam} X)$;

4) $g(x)>0$ для всех $x \in X$.

Свойство 4) следует из того, что $f$ не эквивалентна постоянной, 3) следует из (2.3), а 2) - из теоремы Лебега (см. [2; с. 12]).

Возьмем последовательность $\lambda_{n} \downarrow 0$ и обозначим

$$
A_{n}=\left\{x \in X: g(x)>\lambda_{n}\right\}, \quad k \in \mathbb{N} .
$$

Тогда $A_{n-1} \subset A_{n}$ и $\mu A_{n}<+\infty$. Ряд

$$
\int_{X} g^{p} d \mu=\int_{A_{1}} g^{p} d \mu+\sum_{n=2}^{\infty} \int_{A_{n} \backslash A_{n-1}} g^{p} d \mu
$$

сходится, поэтому существует последовательность $a_{n} \downarrow 0$, для которой

$$
\sum_{n=2}^{\infty} \frac{1}{a_{n}^{p}} \int_{A_{n} \backslash A_{n-1}} g^{p} d \mu<+\infty .
$$

В силу абсолютной непрерывности для каждого $n \in \mathbb{N}$ найдем $\delta_{n}>0$ так, чтобы

$$
\int_{E} g^{p} d \mu<\frac{a_{n}^{p}}{2^{n}} \quad \text { при } \quad \mu E<\delta_{n} .
$$

По теореме Д. Ф. Егорова существует такая последовательность измеримых множеств $E_{n}$, что

$$
E_{n-1} \subset E_{n} \subset A_{n}, \quad \mu\left(A_{n} \backslash E_{n}\right)<\delta_{n+1}
$$

и $g(x, t) / g(x)$ сходится к нулю при $t \rightarrow 0$ равномерно на $E_{n}$. Поэтому существует такая последовательность $t_{n} \downarrow 0$, что

$$
g\left(x, t_{n}\right) \leqslant a_{n+1} g(x) \quad \text { при } \quad x \in E_{n}
$$

$(n \in \mathbb{N})$.

Определим теперь функцию $\psi$ равенством $\psi(1)=a_{0}$ и

$$
\psi(t)=\left\{\begin{array}{l}
a_{n-1} \text { при } t=t_{n}, \\
\text { линейна и непрерывна на }\left[t_{n}, t_{n-1}\right],
\end{array}\right.
$$

где $n \geqslant 1\left(a_{0}=t_{0}=1\right)$. Тогда функция $\psi$ возрастает, $\psi(+0)=0$ и $\psi \in C(0,1]$. 
В качестве искомой функции $\eta$ можно взять выпуклую мажоранту равномерного модуля непрерывности для $\psi$ (см. [20; с.153-154]); тогда $\eta \in \Omega(1) \subset \Omega$ и $\eta(t) \geqslant \psi(t)$.

Докажем, что

$$
\mu\left(X \backslash \bigcup_{k=1}^{\infty} E_{k}\right)=0
$$

В самом деле, ясно, что ${ }^{3}$

$$
\left(\bigcup_{k=1}^{\infty} E_{k}\right)^{c}=\bigcap_{k=1}^{\infty} E_{k}^{c}=\bigcap_{k=1}^{\infty}\left(A_{k}^{c} \cup\left(A_{k} \backslash E_{k}\right)\right) .
$$

Если $x \in \bigcap_{k=1}^{\infty}\left(A_{k}^{c} \cup\left(A_{k} \backslash E_{k}\right)\right)$, то для каждого $k$ либо $x \in A_{k}^{c}$, либо $x \in A_{k} \backslash E_{k}$. Но $x \in A_{k}^{c}$ для бесконечно многих $k$ невозможно (иначе $g(x) \leqslant \lambda_{k}$ для таких $k$ и $g(x)=$ 0 - противоречие), поэтому $x \in A_{k} \backslash E_{k}$, начиная с некоторого $k$. Следовательно,

$$
\left(\bigcup_{k=1}^{\infty} E_{k}\right)^{c} \subset \bigcup_{n=1}^{\infty} \bigcap_{k=n}^{\infty}\left(A_{k} \backslash E_{k}\right)
$$

Но так как для любого $n \in \mathbb{N}$ и всех $m \geqslant n$

$$
\mu\left(\bigcap_{k=n}^{\infty}\left(A_{k} \backslash E_{k}\right)\right) \leqslant \mu\left(A_{m} \backslash E_{m}\right) \leqslant \delta_{m} \rightarrow 0, \quad m \rightarrow \infty,
$$

то

$$
\mu\left(X \backslash \bigcup_{k=1}^{\infty} E_{k}\right) \leqslant \mu\left(\bigcup_{n=1}^{\infty} \bigcap_{k=n}^{\infty}\left(A_{k} \backslash E_{k}\right)\right)=0
$$

и (3.1) доказано.

Пусть $n \in \mathbb{N}$ и $x \in E_{n}$. Тогда $x \in E_{k}$ при всех $k \geqslant n$. Оценим

$$
\sup _{0<t \leqslant 1} \frac{g(x, t)}{\eta(t)}=\sup _{k \geqslant 0} \sup _{t \in\left[t_{k+1}, t_{k}\right]} \frac{g(x, t)}{\eta(t)} \leqslant \max \left\{\max _{0 \leqslant k<n} \frac{g\left(x, t_{k}\right)}{a_{k}}, \sup _{k \geqslant n} \frac{g\left(x, t_{k}\right)}{a_{k}}\right\} \leqslant \frac{g(x)}{a_{n}},
$$

так как $g\left(x, t_{k}\right) \leqslant a_{k+1} g(x)$ при $k \geqslant n$. Итак,

$$
h(x)=\sup _{0<t \leqslant 1} \frac{g(x, t)}{\eta(t)} \leqslant \frac{g(x)}{a_{n}} \quad \text { при } \quad x \in E_{n}
$$

$(n \in \mathbb{N})$.

Используя (3.1), последнее неравенство и очевидное включение

$$
E_{n} \backslash E_{n-1} \subset\left(E_{n} \backslash A_{n-1}\right) \cup\left(A_{n-1} \backslash E_{n-1}\right),
$$

оценим

$$
\int_{X} h^{p} d \mu \leqslant \frac{1}{a_{1}^{p}} \int_{E_{1}} g^{p} d \mu+\sum_{n=2}^{\infty} \frac{1}{a_{n}^{p}} \int_{E_{n} \backslash E_{n-1}} g^{p} d \mu
$$

\footnotetext{
${ }^{3}$ Всюду в работе $E^{c}=X \backslash E-$ дополнение множества $E \subset X$.
} 


$$
\begin{aligned}
& \leqslant \frac{1}{a_{1}^{p}} \int_{E_{1}} g^{p} d \mu+\sum_{n=2}^{\infty} \frac{1}{a_{n}^{p}}\left(\int_{A_{n} \backslash A_{n-1}} g^{p} d \mu+\int_{A_{n-1} \backslash E_{n-1}} g^{p} d \mu\right) \\
& \leqslant \frac{1}{a_{1}^{p}} \int_{E_{1}} g^{p} d \mu+\sum_{n=2}^{\infty} \frac{1}{a_{n}^{p}}\left(\int_{A_{n} \backslash A_{n-1}} g^{p} d \mu+\frac{a_{n}^{p}}{2^{n}}\right)<+\infty
\end{aligned}
$$

и мы получаем утверждение теоремы.

В случае $X=[0,1]^{n}$ утверждение теоремы 2 можно вывести из результатов работы [7], относящихся к связи $\mathscr{N}_{\eta} f$ и $L^{p}$-модуля непрерывности функции $f$.

Теорема 2 показывает, что

$$
L^{p}(X)=\bigcup_{\eta \in \Omega} C_{\eta}^{p}(X)
$$

и, таким образом, мы получаем полную классификацию всех функций из $L^{p}(X)$ по их локальной гладкости с помощью максимальных функций $\mathscr{N}_{\eta}$ или $\mathscr{R}_{\eta}$.

Отметим, что при $p=1$ теорема 2 теряет силу. Действительно, пусть $X$ ограничено и $\operatorname{diam} X=1$; тогда при $\eta \in \Omega$ справедливо неравенство

$$
\mathscr{N}_{\eta} f(x) \geqslant \frac{M f(x)-|f(x)|}{\eta(1)},
$$

а функция Харди-Литтлвуда $M f$ не обязана быть суммируемой для $f \in L^{1}(X)$ (см., например, [21; с. 53]).

Для каждой функции $f \in L^{1}(X)$ мы можем утверждать лишь, что существует функция $\eta \in \Omega$, для которой

$$
\mu\left\{\mathscr{N}_{\eta} f>\lambda\right\} \leqslant \frac{1}{\lambda}\|f\|_{L^{1}(X)}, \quad \lambda>1 .
$$

4. Компактность вложения $C_{\eta}^{p} \subset C_{\sigma}^{q}$. В этом пункте и всюду далее в работе предполагаем, что пространство $X$ является ограниченным (для простоты будем считать, что $\operatorname{diam} X=1$ и $\mu X=1)$. В таком случае из условия (2.1) вытекает, что

$$
\mu B(x, r) \geqslant c r^{\gamma}, \quad x \in X, \quad 0<r \leqslant 1 .
$$

В самом деле, для любого $x \in X$

$$
\mu X=\mu B(x, 1) \leqslant c\left(\frac{1}{r}\right)^{\gamma} \mu B(x, r) .
$$

Поэтому условие (2.4) леммы 1 выполнено. Этим обосновано использование леммы 1 ниже в доказательстве теоремы 3.

Кроме того, нам понадобится следующее простое неравенство:

$$
\|f\|_{L^{p}(X)} \leqslant \sigma(1)\left\|\mathscr{R}_{\sigma} f\right\|_{L^{p}(X)}+\|f\|_{L^{1}(X)} .
$$

Теорема 3. Пусть $1<p<q<\infty, \sigma \in \Omega, \eta$ определяется равенством (1.5). Тогда если $\sigma_{0} \in \Omega$ такова, что

$$
\lim _{r \rightarrow+0} \frac{\sigma(r)}{\sigma_{0}(r)}=0
$$

то вложения $C_{\eta}^{p}(X) \subset C_{\sigma_{0}}^{q}(X)$ u $S_{\eta}^{p}(X) \subset S_{\sigma_{0}}^{q}(X)$ компактны. 
ДокАЗАтеЛьство. Пусть $K$ - ограниченное множество в $C_{\eta}^{p}(X)$ и

$$
A(K)=\sup _{f \in K}\left(\|f\|_{L^{p}(X)}+\left\|\mathscr{N}_{\eta} f\right\|_{L^{p}(X)}\right)<+\infty .
$$

Докажем, что $K$ является вполне ограниченным в $C_{\sigma_{0}}^{q}(X)$ - зададим $\varepsilon>0$ и построим для него конечную $\varepsilon$-сеть.

Введем обозначение

$$
\eta_{0}(r)=\sigma_{0}(r) r^{\gamma(1 / p-1 / q)}
$$

Тогда в силу (4.3)

$$
\omega(t)=\sup _{0<r \leqslant t} \frac{\eta(r)}{\eta_{0}(r)}=\sup _{0<r \leqslant t} \frac{\sigma(r)}{\sigma_{0}(r)} \rightarrow 0, \quad t \rightarrow+0 .
$$

Выберем $\delta>0$ таким образом, чтобы

$$
\omega(\delta)<\frac{\varepsilon}{A(K)} .
$$

В частности, из (4.4) следует, что $K$ ограничено в $L^{p}(X)$ и в силу левого неравенства (2.9) выполнено (2.5). По лемме 1 множество $K$ является вполне ограниченным и для него существует конечная $\varepsilon \eta_{0}(\delta)$-сеть $\left\{f_{i}\right\}_{i=1}^{m}$ в $L^{p}(X)$, причем можно считать, что $f_{i} \in K, i=1, \ldots, m$.

Покажем, что $\left\{f_{i}\right\}_{i=1}^{m}$ является сє-сетью для $K$ в $C_{\sigma_{0}}^{q}(X)$. Пусть $f \in K$ и $1 \leqslant i \leqslant m$ выбрано так, что

$$
\|h\|_{L^{p}(X)}<\eta_{0}(\delta) \varepsilon, \quad h=f-f_{i} .
$$

Докажем, что

$$
\|h\|_{C_{\sigma_{0}}^{q}(X)}<c \varepsilon .
$$

Сначала отметим, что так как $f, f_{i} \in K$, то

$$
\left\|\mathscr{N}_{\eta} h\right\|_{L^{p}(X)} \leqslant 2 A(K)
$$

(см. (4.4)). Далее, для почти всех $x \in X$

$$
\sup _{B \in \mathscr{B}, r(B) \geqslant \delta} \frac{1}{\eta_{0}(r(B))} f_{B}|h(x)-h(y)| d \mu(y) \leqslant \frac{2 M h(x)}{\eta_{0}(\delta)},
$$

так как $|h(x)| \leqslant M h(x)$ почти всюду на $X$. Кроме того (см. (4.6)),

$$
\begin{aligned}
\sup _{B \in \mathscr{B}(x, \delta)} \frac{1}{\eta_{0}(r(B))} f_{B}|h(x)-h(y)| d \mu & =\sup _{B \in \mathscr{B}(x, \delta)} \frac{\eta(r(B))}{\eta_{0}(r(B))} \cdot \frac{1}{\eta(r(B))} f_{B}|h(x)-h(y)| d \mu \\
& \leqslant \omega(\delta) \sup _{B \in \mathscr{B}(x, \delta)} \frac{1}{\eta(r(B))} f_{B}|h(x)-h(y)| d \mu(y) \\
& \leqslant \omega(\delta) \mathscr{N}_{\eta} h(x) .
\end{aligned}
$$

Из последних двух неравенств вытекает оценка

$$
\left\|\mathscr{N}_{\eta_{0}} h\right\|_{L^{p}(X)} \leqslant \frac{2\|M h\|_{L^{p}(X)}}{\eta_{0}(\delta)}+\omega(\delta)\left\|\mathscr{N}_{\eta} h\right\|_{L^{p}(X)} .
$$


Кроме того, из (4.2), (2.9) и неравенства (1.6) из теоремы 1 (примененной к функциям $\sigma_{0}$ и $\eta_{0}$ из $\left.(4.5)\right)$, получаем $\|h\|_{C_{\sigma_{0}}^{q}(X)} \leqslant c\|h\|_{C_{\eta_{0}}^{p}(X)}$.

Отсюда и из (4.11), (4.7), (4.8) и (4.10) вытекает неравенство

$$
\|h\|_{C_{\sigma_{0}}^{q}(X)} \leqslant c\left(\frac{\|h\|_{L^{p}(X)}}{\eta_{0}(\delta)}+\omega(\delta)\left\|\mathscr{N}_{\eta} h\right\|_{L^{p}(X)}\right)<c \varepsilon
$$

и (4.9) доказано.

Компактность вложения $S_{\eta}^{p}(X) \subset S_{\sigma_{0}}^{q}(X)$ следует из уже доказанного и из неравенств (2.8) и (2.10), так как функция $\eta$ из (1.5) удовлетворяет условию Бари (2.11). Теорема 3 доказана.

Далее мы установим, что условие (4.3) является необходимым для компактности рассматриваемых вложений во многих ситуациях, но это можно сделать лишь при некоторых дополнительных предположениях относительно параметров.

Прежде всего потребуем более сильного ограничения на связь меры $\mu$ и квазиметрики $d$. Именно, пусть

$$
c^{-1} r^{\gamma} \leqslant \mu B(x, r) \leqslant c r^{\gamma}, \quad x \in X, \quad 0<r \leqslant 1 .
$$

В таком случае $\mu$ часто называют $\gamma$-регулярной, а $(X, d, \mu)-\gamma$-множеством [22]. Неравенство (4.1) показывает, что по сравнению с условием удвоения (2.1) новым в (4.13) является лишь правое неравенство.

Кроме того, вместо $\eta \in \Omega$ будем требовать $\eta \in \Omega(1)$. Наконец, предположим, что $d$ является метрикой.

Теорема 4. Пусть $1<p<q<\infty$, мера $\mu$ удовлетворяет условию (4.13), $\eta \in$ $\Omega(1), \sigma$ определяется равенством (1.5). Тогда если $\sigma_{0} \in \Omega$, то из компактности вложения $C_{\eta}^{p}(X) \subset C_{\sigma_{0}}^{q}(X)$ следует $(4.3)$.

ДокАЗАТЕЛЬСтво. Покажем, что если

$$
\limsup _{r \rightarrow+0} \frac{\sigma(r)}{\sigma_{0}(r)}>0
$$

то существует ограниченная последовательность $\left\{f_{k}\right\} \subset C_{\eta}^{p}(X)$, из которой нельзя выделить подпоследовательность, сходящуюся в $C_{\sigma_{0}}^{q}(X)$. При этом мы будем использовать конструкцию из доказательства предложения 3.2 в [7].

В силу (1.5) и (4.14) найдется такая последовательность положительных чисел $\rho_{k}>0$, что

$$
\frac{\eta\left(\rho_{k}\right)}{\sigma_{0}\left(\rho_{k}\right) \rho_{k}^{\gamma(1 / p-1 / q)}} \geqslant c>0 .
$$

Далее, используя условие регулярности (4.13), легко построить последовательность непересекающихся шаров $B\left(x_{k}, t_{k}\right) \subset X, t_{k} \downarrow 0$. Для $k=1,2 \ldots$ положим $r_{k}=\rho_{j}$, где $j=\min \left\{i: t_{k} \geqslant 2 \rho_{i}\right\}$. Тогда

$$
B\left(x_{i}, 2 r_{i}\right) \cap B\left(x_{k}, 2 r_{k}\right)=\varnothing, \quad i \neq k,
$$


и справедливы неравенства

$$
\frac{\eta\left(r_{k}\right)}{\sigma_{0}\left(r_{k}\right) r_{k}^{\gamma(1 / p-1 / q)}} \geqslant c>0
$$

Пусть $B_{k} \equiv B\left(x_{k}, r_{k}\right)$ и

$$
f_{k}(x)=H_{k}\left(1-\frac{d\left(x, x_{k}\right)}{r_{k}}\right) \chi_{B_{k}}(x), \quad H_{k}=\eta\left(r_{k}\right) r_{k}^{-\gamma / p} .
$$

Покажем, что последовательность $\left\{f_{k}\right\}$ ограничена в $C_{\eta}^{p}(X)$. Сначала получим равномерную оценку для $\mathscr{R}_{\eta} f_{k}(x)$. Пусть $x \in X$ и $B \in \mathscr{B}(x)$. Если $r_{k} \leqslant r(B)$, то

$$
\frac{\left|f_{k}(x)-\left[f_{k}\right]_{B}\right|}{\eta(r(B))} \leqslant \frac{2 H_{k}}{\eta(r(B))} \leqslant \frac{2 H_{k}}{\eta\left(r_{k}\right)} .
$$

Если же $r_{k}>r(B)$, то из неравенств

$$
\left|f_{k}(x)-f_{k}(y)\right| \leqslant H_{k} \frac{\left|d\left(x, x_{k}\right)-d\left(y, x_{k}\right)\right|}{r_{k}} \leqslant \frac{H_{k}}{r_{k}} d(x, y) \leqslant \frac{2 H_{k}}{r_{k}} r(B), \quad y \in B,
$$

и из условия $\eta \in \Omega(1)$ следует, что

$$
\frac{\left|f_{k}(x)-\left[f_{k}\right]_{B}\right|}{\eta(r(B))} \leqslant \frac{1}{\eta(r(B))} f_{B}\left|f_{k}(x)-f_{k}(y)\right| d \mu(y) \leqslant \frac{2 H_{k}}{\eta\left(r_{k}\right)} .
$$

Таким образом,

$$
\mathscr{R}_{\eta} f_{k}(x) \leqslant \frac{2 H_{k}}{\eta\left(r_{k}\right)} \quad \text { при } \quad x \in X .
$$

Мы будем использовать эту оценку при $d\left(x, x_{k}\right) \leqslant 2 r_{k}$.

Для $d\left(x, x_{k}\right)>2 r_{k}$ (4.17) можно улучшить - тогда $x \notin B_{k}$ и для любого шара $B \in \mathscr{B}(x)$

$$
\frac{\left|f_{k}(x)-\left[f_{k}\right]_{B}\right|}{\eta(r(B))}=\frac{\left|\left[f_{k}\right]_{B}\right|}{\eta(r(B))} \leqslant \frac{H_{k}}{\eta(r(B))} \cdot \frac{\mu\left(B \cap B_{k}\right)}{\mu B} .
$$

Если $B \cap B_{k} \neq \varnothing$ и $y \in B \cap B_{k}$, то

$$
d\left(x, x_{k}\right) \leqslant d\left(x, y_{0}\right)+d\left(y_{0}, y\right)+d\left(y, x_{k}\right) \leqslant 2 r(B)+r_{k}
$$

$\left(y_{0}\right.$ - центр $\left.B\right)$ и $2 r(B) \geqslant d\left(x, x_{k}\right)-r_{k} \geqslant d\left(x, x_{k}\right) / 2$. Поэтому

$$
\frac{\left|f_{k}(x)-\left[f_{k}\right]_{B}\right|}{\eta(r(B))} \leqslant c \frac{H_{k}}{\eta(r(B))} \cdot \frac{\mu B_{k}}{\mu B} \leqslant c \frac{H_{k}}{\eta\left(d\left(x, x_{k}\right)\right)}\left(\frac{r_{k}}{d\left(x, x_{k}\right)}\right)^{\gamma} .
$$

Таким образом,

$$
\mathscr{R}_{\eta} f_{k}(x) \leqslant c \frac{H_{k}}{\eta\left(d\left(x, x_{k}\right)\right)}\left(\frac{r_{k}}{d\left(x, x_{k}\right)}\right)^{\gamma} \quad \text { при } \quad d\left(x, x_{k}\right)>2 r_{k} .
$$

С помощью (4.18) и (4.17) оценим теперь норму

$$
\left\|\mathscr{R}_{\eta} f_{k}\right\|_{L^{p}(X)}^{p}=\int_{X \backslash B\left(x_{k}, 2 r_{k}\right)}\left[\mathscr{R}_{\eta} f_{k}\right]^{p} d \mu+\int_{B\left(x_{k}, 2 r_{k}\right)}\left[\mathscr{R}_{\eta} f_{k}\right]^{p} d \mu \equiv I_{1}+I_{2} .
$$


Для оценки $I_{1}$ воспользуемся неравенством (4.18):

$$
\begin{aligned}
I_{1} & \leqslant c r_{k}^{\gamma p} H_{k}^{p} \int_{d\left(x, x_{k}\right)>2 r_{k}} \frac{d \mu(x)}{\left(\eta\left(d\left(x, x_{k}\right)\right)\right)^{p}\left(d\left(x, x_{k}\right)\right)^{\gamma p}} \\
& =c r_{k}^{\gamma p} H_{k}^{p} \sum_{i=0}^{m} \int_{2^{i} r_{k}<d\left(x, x_{k}\right) \leqslant 2^{i+1} r_{k}} \frac{d \mu(x)}{\left(\eta\left(d\left(x, x_{k}\right)\right)\right)^{p}\left(d\left(x, x_{k}\right)\right)^{\gamma p}} \\
& =c r_{k}^{\gamma p} H_{k}^{p} \sum_{i=0}^{m}\left[\eta\left(2^{i} r_{k}\right)\left(2^{i} r_{k}\right)^{\gamma}\right]^{-p}\left(2^{i+1} r_{k}\right)^{\gamma} \\
& =c r_{k}^{\gamma p} H_{k}^{p}\left[\eta\left(r_{k}\right)\right]^{-p} r_{k}^{-\gamma p+\gamma} \sum_{i=0}^{m} 2^{i \gamma(1-p)} \leqslant c
\end{aligned}
$$

(здесь $\left.m=\left[\log _{2} 1 / r_{k}\right]+1\right)$.

Для оценки $I_{2}$ применим (4.17):

$$
I_{2} \leqslant\left[\frac{2 H_{k}}{\eta\left(r_{k}\right)}\right]^{p} \mu\left(2 B_{k}\right) \leqslant c .
$$

Итак, $\left\|\mathscr{R}_{\eta} f_{k}\right\|_{L_{p}(X)} \leqslant c$. Кроме того, очевидно, что $\left\|f_{k}\right\|_{L_{p}(X)} \leqslant c \eta\left(r_{k}\right) \leqslant c$. Следовательно, в силу неравенств $(2.9)$ и (2.3) последовательность $f_{k}$ ограничена в $C_{\eta}^{p}(X)$.

С другой стороны, с помощью условия (4.13) можно подобрать $\delta \in(0,1 / 2)$ так, чтобы $\mu\left(B_{k} \backslash 2 \delta B_{k}\right) \geqslant \mu B_{k} / 2$ для всех $k$. Следовательно, для любых $k, i \in \mathbb{N}$ и $x \in \delta B_{k}$

$$
\begin{aligned}
\mathscr{N}_{\sigma_{0}}\left(f_{k}-f_{i}\right)(x) & \geqslant \frac{1}{\sigma_{0}\left(r_{k}\right)} f_{B_{k}}\left|\left[f_{k}(x)-f_{k}(y)\right]-\left[f_{i}(x)-f_{i}(y)\right]\right| d \mu(y) \\
& =\frac{1}{\sigma_{0}\left(r_{k}\right) \mu B_{k}} \int_{B_{k} \backslash 2 \delta B_{k}}\left|\left[f_{k}(x)-f_{k}(y)\right]\right| d \mu(y) \\
& =\frac{H_{k}}{\sigma_{0}\left(r_{k}\right) \mu B_{k}} \int_{B_{k} \backslash 2 \delta B_{k}}\left[\frac{d\left(y, x_{k}\right)-d\left(x, x_{k}\right)}{r_{k}}\right] d \mu(y) \\
& \geqslant c \frac{H_{k}}{\sigma_{0}\left(r_{k}\right)} \cdot \frac{\mu\left(B_{k} \backslash 2 \delta B_{k}\right)}{\mu B_{k}} \geqslant c r_{k}^{-\gamma / q}
\end{aligned}
$$

(см. неравенство (4.15)). Отсюда получаем

$$
\left\|f_{k}-f_{i}\right\|_{C_{\sigma_{0}}^{q}(X)}^{q} \geqslant \int_{\delta B_{k}}\left[\mathscr{N}_{\sigma_{0}}\left(f_{k}-f_{i}\right)\right]^{q} d \mu \geqslant c>0
$$

и из $f_{k}$ нельзя выделить подпоследовательность, сходящуюся в $C_{\sigma_{0}}^{q}(X)$. Теорема доказана.

Сделаем теперь ряд замечаний по поводу формулировки теоремы 4.

Прежде всего отметим, что утверждение теоремы 4 содержательно лишь при $1 / p-1 / q<1 / \gamma$. Если это неравенство не выполнено, то условия (4.3) и $\sigma_{0} \in \Omega$ в ее формулировке несовместимы.

Далее, дополнительные ограничения на $\mu$ и $\eta$ в теореме 4 по сравнению с теоремой 3 являются существенными - без них утверждение теоремы 4 становится неверным. 
Во-первых, если взять $X=[0,1]^{n}$ с евклидовой метрикой и мерой Лебега, а для $\eta$ выполнено (1.7), то (см. замечание 2) класс $C_{\eta}^{p}\left([0,1]^{n}\right)$ состоит только из постоянных (с точностью до эквивалентности) функций. Поэтому вложение $C_{\eta}^{p}\left([0,1]^{n}\right) \subset$ $C_{\sigma}^{q}\left([0,1]^{n}\right)$ в любой класс $C_{\sigma}^{q}\left([0,1]^{n}\right)$ компактно. Взяв здесь $\eta(r)=r^{1+\varepsilon}(\varepsilon>0)$ получаем, что требование $\eta \in \Omega(1)$ в теореме 4 нельзя заменить любым условием $\eta \in \Omega(1+\varepsilon), \varepsilon>0$.

Во-вторых, в теореме 4 условие регулярности (4.13) нельзя заменить условием (2.1) - тогда (2.1) может выполняться с некоторым $\widetilde{\gamma}<\gamma$ (см. замечание 5). По теореме 3 достаточным условием для компактности вложения $C_{\eta}^{p}(X) \subset C_{\sigma_{0}}^{q}(X)$ будет требование

$$
\lim _{r \rightarrow+0} \frac{\eta(r) r^{\tilde{\gamma}(1 / q-1 / p)}}{\sigma_{0}(r)}=0,
$$

более слабое по сравнению с (4.3).

5. Компактность вложения $C_{\eta}^{p} \subset L^{q}$. Из теоремы 1 легко выводится следующее утверждение.

TEOPEMA 5. Пусть $1<p<q<\infty, \eta(r)=r^{\gamma(1 / p-1 / q)}$. Тогда

$$
\|f\|_{L^{q}(X)} \leqslant c\|f\|_{C_{\eta}^{p}(X)} .
$$

В частности, $C_{\eta}^{p}(X) \subset L^{q}(X)$.

ДокаЗАТЕЛЬСтво. Пусть $x_{0} \in X$; тогда $X \subset B_{0}=B\left(x_{0}, 1\right)$. Применим теорему 1 в случае $\sigma(r) \equiv 1$ (см. замечание 4). Отсюда в силу (2.9) и (1.6) получаем

$\|f\|_{L^{q}(X)} \leqslant\left\|f-f_{B_{0}}\right\|_{L^{q}(X)}+\left|f_{B_{0}}\right| \leqslant \sigma(1)\left\|\mathscr{R}_{\sigma} f\right\|_{L^{q}(X)}+(\mu X)^{1-1 / p}\|f\|_{L^{p}(X)} \leqslant c\|f\|_{C_{\eta}^{p}(X)}$.

Вопрос о компактности вложения из теоремы 5 решает следующее утверждение.

TeOpema 6. Пусть $1<p<q<\infty u \eta \in \Omega$,

$$
\lim _{r \rightarrow+0} \eta(r) r^{\gamma(1 / q-1 / p)}=0 .
$$

Тогда вложение $C_{\eta}^{p}(X) \subset L^{q}(X)$ компактно.

ДокАЗАТЕЛЬСтво проходит так же, как и в теореме 3. Укажем необходимые изменения.

Пусть $\eta_{0}(r)=r^{\gamma(1 / p-1 / q)}$. Вместо (4.6) полагаем

$$
\omega(t)=\sup _{0<r \leqslant t} \frac{\eta(r)}{\eta_{0}(r)}
$$

и выбираем $\delta>0$ так, чтобы выполнялось (4.7). После этого находим $\varepsilon \eta_{0}(\delta)$-сеть для $K$ в $L^{p}(X)$ и повторим рассуждения из теоремы 3 , применяя теорему 5 вместо теоремы 1. Тогда вместо (4.12) получим неравенство

$$
\|h\|_{L^{q}(X)} \leqslant c\left(\frac{\|h\|_{L^{p}(X)}}{\eta_{0}(\delta)}+\omega(\delta)\left\|\mathscr{N}_{\eta} h\right\|_{L^{p}(X)}\right)<c \varepsilon .
$$

Как и теорема 4 в предыдущем пункте, теорема 6 неулучшаема при тех же дополнительных ограничениях. В следующей теореме, как и в теореме 4, предполагаем, что $d$ является метрикой. 
Теорема 7. Пусть $1<p<q<\infty$, мера $\mu$ удовлетворяет условию (4.13), $\eta \in$ $\Omega(1)$. Тогда из компактности вложения $C_{\eta}^{p}(X) \subset L^{q}(X)$ следует (5.1).

ДоКАЗАТЕЛЬСТво. Повторим рассуждения из доказательства теоремы 4 , взяв в них $\sigma_{0}(r) \equiv 1$. Тогда получим, что последовательность (4.16) ограничена в $C_{\eta}^{p}(X)$.

После этого, выбирая $0<\delta<1 / 4$ так, чтобы $\mu\left(B_{k} \backslash 2 \delta B_{k}\right) \geqslant \mu B_{k} / 2$ для всех $k$, получаем

$$
\begin{aligned}
\left\|f_{k}-f_{i}\right\|_{L^{q}(X)}^{q} & \geqslant \int_{B_{k}}\left|f_{k}\right|^{q} d \mu \geqslant \int_{B_{k} \backslash 2 \delta B_{k}}\left[H_{k}\left(1-\frac{d\left(x, x_{k}\right)}{r_{k}}\right)\right]^{q} d \mu(x) \\
& \geqslant c \mu B_{k} H_{k}^{q}=\left[\eta\left(r_{k}\right) r_{k}^{\gamma(1 / q-1 / p)}\right]^{q} \geqslant c>0
\end{aligned}
$$

для всех $k, i \in \mathbb{N}$.

$\mathrm{K}$ теореме 7 сделаем следующие замечания. Во-первых, она содержательна лишь при $1 / p-1 / q<1 / \gamma-$ без этого неравенства условия $\eta \in \Omega(1)$ и (5.1) в формулировке теоремы несовместимы.

Во-вторых, в формулировке теоремы 7 нельзя отказаться от условия регулярности (4.13), заменив его условием (2.1). Кроме того, условие $\eta \in \Omega(1)$ нельзя заменить на $\eta \in \Omega(1+\varepsilon)$, где $\varepsilon>0$. Причины для этого такие же, как и указанные выше для теоремы 4 .

\section{СПИСОК ЦИТИРОВАННОЙ ЛИТЕРАТУРЫ}

[1] R. R. Coifman, G. Weiss, Analyse Harmonique non-commutative sur certains espaces homogénes. Étude de certaines intégrales singulières, Lecture Notes in Math., 242, Springer-Verlag, Berlin, 1971.

[2] J. Heinonen, Lectures on Analysis on Metric Spaces, Universitext, Springer-Verlag, Berlin, 2001.

[3] A. P. Calderón, "Estimates for singular integral operators in terms of maximal functions", Studia Math., 44 (1972), 563-582.

[4] A. P. Calderón, R. Scott, "Sobolev type inequalities for $p>0$ ", Studia Math., 62:1 (1978), $75-92$.

[5] R. A. DeVore, R. C. Sharpley, Maximal Functions Measuring Smoothness, Mem. Amer. Math. Soc., 47, no. 293, Amer. Math. Soc., Providence, RI, 1984.

[6] В. И. Коляда, "Оценки максимальных функций, связанных с локальной гладкостью", Докл. АН СССР, 293:3 (1987), 534-537.

[7] V. I. Kolyada, "Estimates of maximal functions measuring local smoothness", Anal. Math., 25:4 (1999), 277-300.

[8] К. И. Осколков, "Аппроксимативные свойства суммируемых функций на множествах полной меры”, Матем. сб., 103:4 (1977), 563-589.

[9] P. Hajłasz, "Sobolev spaces on an arbitrary metric space", Potential Anal., 5:4 (1996), 403-415.

[10] И. А. Иванишко, "Оценки максимальных функций Кальдерона-Коляды на пространствах однородного типа", Тр. ин-та матем. НАН Беларуси, 12:1 (2004), 64-67.

[11] Л.К. Эванс, Р. Ф. Гариепи, Теория меры и тонкие свойства функиий, Университетская серия, 9, Научная книга, Новосибирск, 2002.

[12] Э. Либ, М. Лосс, Анализ, Университетская серия, 1, Научная книга, Новосибирск, 1998. 
[13] A. Jonsson, "Haar wavelets of higher order on fractals and regularity of functions", J. Math. Anal. Appl., 290:1 (2004), 86-104.

[14] A. Kałamajska, "On compactness of embedding for Sobolev spaces defined on metric spaces", Ann. Acad. Sci. Fenn. Math., 24:1 (1999), 123-132.

[15] В. И. Коляда, Теоремъ вложения и метрические свойства функиий, Дис. ... докт. физ.-матем. наук, Одесса, ОГУ, 1986.

[16] И. А. Иванишко, "Обобщенные классы Соболева на метрических пространствах с мерой", Матем. заметки, 77:6 (2005), 937-941.

[17] D. Yang, "New characterizations of Hajłasz-Sobolev spaces on metric spaces", Sci. China Ser. A, 46:5 (2003), 675-689.

[18] В.Г. Кротов, "Весовые $L^{p}$-неравенства для шарп-максимальных функций", Докл. PAH, 404:2 (2005), 155-158.

[19] В.Г. Кротов, "Весовые $L^{p}$-неравенства для шарп-максимальных функций на метрических пространствах с мерой”, Изв. НАН Армении. Сер. матем., 41:2 (2006), 25-42.

[20] В. К. Дзядык, Введение в теорию равномерного приближения функиий полиномами, Наука, М., 1977.

[21] И. М. Стейн, Сингулярные интегралы и дифференииальные свойства функиий, Мир, M., 1973.

[22] H. Triebel, Theory of Function Spaces. III, Monogr. Math., 100, Birkhäuser-Verlag, Basel, 2006.

\section{И. А. Иванишко}

Белорусский государственный университет, г. Минск

E-mail: ivanishko@bsu.by

\section{В. Г. Кротов}

Белорусский государственный университет, г. Минск

E-mail: krotov@bsu.by
Поступило

06.04.2009 